\title{
MECANISMOS DE AÇÃO DOS PRINCIPAIS ANTIOXIDANTES UTILIZADOS NA CRIOPRESERVAÇÃO ESPERMÁTICA DE GARANHÕES
}

\author{
Luis Fernando Merces Chaves Silva ${ }^{1}$ \\ Endrigo Adonis Braga de Araujo ${ }^{1}$ \\ Sidnei Nunes de Oliveira ${ }^{1}$ \\ Felipe Morales Dalanezi ${ }^{1}$ \\ Luiz Roberto Pena de Andrade Júnior ${ }^{1}$ \\ Fabiana Ferreira de Souza ${ }^{1}$ \\ André Maciel Crespilho ${ }^{2}$ \\ José Antonio Dell'Aqua Junior ${ }^{1}$ \\ Frederico Ozanam Papa ${ }^{3}$
}

\begin{abstract}
RESUMO
O emprego da criopreservação espermática na reprodução equina representou um grande avanço para o desenvolvimento da espécie. Entretanto a utilização de biotecnicas como a refrigeração e congelação espermática imprime sobre as células danos físicos e químicos que resultam em elevação nos níveis de espécies reativas do oxigênio (EROs), estresse oxidativo e injúrias irreversíveis aos espermatozoides. Visando amenizar as consequências do estresse oxidativo, diversas substancias com propriedades antioxidantes são adicionadas aos meios diluidores durante a criopreservação, apesar disto, na maioria das vezes o mecanismo de ação desencadeado por estes antioxidantes não é bem esclarecido. Diante disto, objetivou-se com o presente estudo referenciar os principais agentes antioxidantes utilizados na criopreservação de garanhões e seus mecanismos de ação.
\end{abstract}

Palavras-chave: radicais livres, antioxidantes, sêmen, equino.

\section{KEY OF MECHANISMS OF ACTION ANTIOXIDANTS USED IN CRYOPRESERVATION SPERM STALLIONS}

\begin{abstract}
The use of sperm cryopreservation in equine reproduction represented a major breakthrough for the development of the species. However the use of biotechnologies such as refrigeration and freezing sperm prints on the physical and chemical damage cells that result in increased levels of reactive oxygen species (ROS), oxidative stress and irreversible injury to sperm. Aiming to mitigate the consequences of oxidative stress, different substances with antioxidant properties are added to extenders for cryopreservation, despite this, most of the time the mechanism of action triggered by these antioxidants is not well understood. In view of this, the aim with this study reference the main antioxidants used in cryopreservation stallions and their mechanisms of action.
\end{abstract}

Keywords: free radicals, antioxidants, semen, equine.

\footnotetext{
${ }^{1}$ Mestrando em Biotecnologia Animal. Universidade Estadual Paulista "Julio de Mesquita Filho" - FMVZ-UNESPBotucatu. Contato principal para correspondência.

${ }^{2}$ Universidade de Santo Amaro - UNISA - SP.

${ }^{3}$ Prof.Titular do Departamento de Reprodução Animal. FMVZ-UNESP-Botucatu.
}

Silva LFMC, Araujo EAB, Oliveira SN, Dalanezi FM, Andrade Junior LRP, Souza FF, Crespilho AM. et al. Mecanismos de ação dos principais antioxidantes utilizados na criopreservação espermática de garanhões. Vet. e Zootec. 2017 Set.; 24(3): 418-434. 


\section{MECANISMOS DE ACCIÓN DE LOS ANTIOXIDANTES QUE SE UTILIZAN EN CRIOPRESERVACIÓN ESPERMÁTICA DE CABALLOS RESUMEN}

El uso de la criopreservación de espermatozoides en reproducción equina representa un avance importante para el desarrollo de la especie. Sin embargo, el uso de las biotecnologías, como la refrigeración y congelación de esperma impresiones sobre las células de daños físicos y químicos que resultan en un aumento de los niveles de especies reactivas de oxígeno (ROS), estrés oxidativo y el daño irreversible a los espermatozoides. Con el objetivo de mitigar las consecuencias del estrés oxidativo, se añaden diferentes sustancias con propiedades antioxidantes de diluyentes para la criopreservación, a pesar de esto, la mayoría de las veces el mecanismo de acción provocada por estos antioxidantes no se entiende bien. En vista de esto, el objetivo de este estudio de referencia los principales antioxidantes utilizados en los sementales de criopreservación y sus mecanismos de acción.

Palabras clave: los radicales libres, los antioxidantes, el semen, caballo.

\section{INTRODUÇÃO}

A utilização do sêmen criopreservado é uma prática amplamente utilizada na maioria das raças dentro da espécie equina, realizando-se biotécnicas como refrigeração e congelação de sêmen, que permitem a manutenção da viabilidade celular por tempo prolongado pela preservação pelo frio (1).

Diferente de outras espécies, os equinos geralmente não são selecionados de acordo com sua capacidade reprodutiva, levando-se em consideração, na maioria das vezes, exclusivamente a sua performance atlética ou características fenotípicas, em detrimento de sua qualidade seminal, acarretando em um grande número de garanhões com sêmen sensível à criopreservação (2).

Um dos fatores relacionados à queda na viabilidade dos espermatozoides equinos durante os processos de refrigeração e congelação é a alta predisposição destas células a sofrerem danos oxidativos resultantes da elevação nos níveis de espécies reativas do oxigênio (EROs) $(3,4)$. Em pequenas quantidades, as EROs desencadeiam eventos de sinalização celular e regulação da maturação, capacitação, hiperativação espermática, reação acrossomal e a união entre espermatozoide e oócito (5-7).

Entretanto, quando em elevadas concentrações são prejudiciais, levando ao estresse oxidativo, induzindo peroxidação lipídica, fragmentação do ácido desoxirribonucleico (DNA) e apoptose precoce das células (8). Neste contexto, a adição de agentes antioxidantes aos meios diluidores visa balancear a concentração de EROs objetivando reduzir os danos promovidos nos espermatozoides.

Diversos antioxidantes existentes têm sido utilizados como suplementos em meios diluidores de sêmen equino, dentre eles a superóxido dismutase (9), glutationa peroxidase (10), catalase (11,12), $\alpha$-tocoferol (13-15), ácido ascórbico $(11,14)$, butil-hidroxitolueno (BHT) $(2,12)$ e quercetina $(16,17)$. Esta revisão bibliográfica visa atualizar os conhecimentos sobre a aplicação e os mecanismos de ação de alguns antioxidantes utilizados na criopreservação de espermatozoides equinos.

\section{METABOLISMO ESPERMÁTICO E FORMAÇÃO DAS ERO}

Os espermatozoides são células altamente especializadas com a função de fertilizar e transferir o material genético masculino ao gameta feminino (18). Para se locomover e 
desempenhar sua função, as células espermáticas dos equinos têm como principal fonte de energia as moléculas de adenosina trifosfato (ATP), resultantes do metabolismo da glicose (19).

Durante o metabolismo espermático, a molécula de glicose e seus substratos passarão por etapas importantes, como a glicólise, passando pelo complexo piruvato-desidrogenase e ciclo do ácido cítrico (20). Ao longo destas etapas são formadas coenzimas reduzidas como as nicotinamida-adenina-dinucleotídeo reduzida (NADH) e flavina-adenina-dinucleotídeo reduzida $\left(\mathrm{FADH}_{2}\right)$, e cada uma têm a função de carrear um par de elétrons, liberados durante as reações de desidrogenação ocorridas no metabolismo da glicose, direcionando-os à cadeia de transporte de elétrons $(20,21)$.

A cadeia de transporte de elétrons é uma etapa regida por reações que ocorrem nos diversos complexos enzimáticos inclusos na membrana interna da mitocôndria. Ao todo são quatro complexos (I, II, III e IV) compostos por proteínas que têm a função de permitir e auxiliar no transporte de elétrons dentro da cadeia, resultando na transferência de energia para fosforilação oxidativa e síntese de ATP (22).

Os NADH atuando no complexo I e os FADH2 no complexo II, vão transferir seu par de elétrons para outra coenzima a ubiquinona $(\mathrm{Q})$ se convertendo em ubiquinol $\left(\mathrm{QH}_{2}\right)$, que por sua vez tem a função de carrear os elétrons em direção a moléculas de citocromos $\mathrm{C}$ (Cit C) no complexo III, ao chegar no complexo IV os Cit $\mathrm{C}$ passam seus elétrons ao oxigênio $\left(\mathrm{O}_{2}\right)$, aceptor final dos elétrons na cadeia, formando moléculas de água $\left(\mathrm{H}_{2} \mathrm{O}\right)(21)$.

Durante a transferência do par de elétrons dos $\mathrm{NADH}_{\text {e }} \mathrm{FADH}_{2}$ às $\mathrm{Q}$, bem como pela transferência dos elétrons dos $\mathrm{QH}_{2}$ aos Cit $\mathrm{C}$, ocorre a formação do radical intermediário, o ânion semiquinona $\left(\mathrm{Q}^{-}\right)$que ocasionalmente é capaz de transferir este elétron desemparelhado a uma molécula de $\mathrm{O}_{2}$ circulante, promovendo a formação da ERO radical superóxido $\left(\mathrm{O}_{2}{ }^{-}\right)$ (23) (Figura 1).

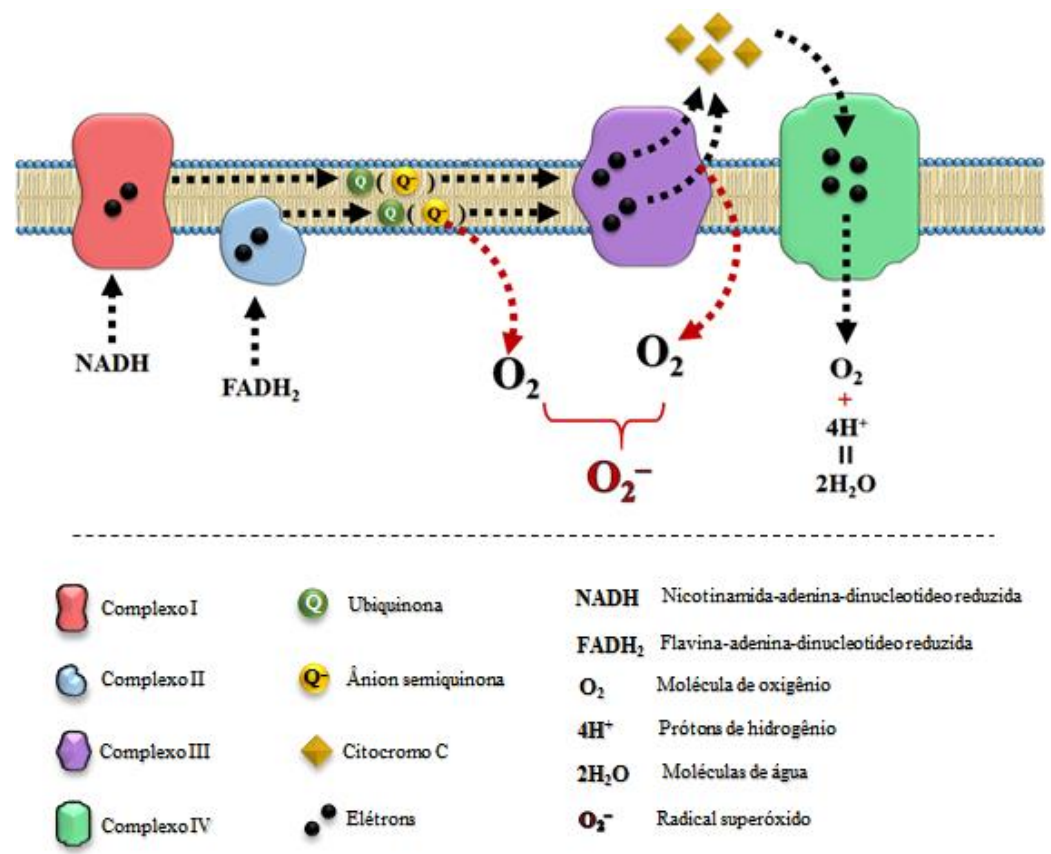

Figura 1. Formação do radical superóxido $\left(\mathrm{O}_{2}^{-}\right)$durante a cadeia de transporte de elétrons.

Este aparentemente é o principal mecanismo responsável pela produção das EROs e sua ocorrência é mais comum nos complexos I e III, atribuindo-se uma probabilidade de efusão de elétrons, duas vezes maior ao complexo III quando comparado ao complexo I (24). 


\section{PRINCIPAIS EROs}

As principais EROs conhecidas resultantes do metabolismo espermático são o $\mathrm{O}_{2}{ }^{-}$, o peróxido de hidrogênio $\left(\mathrm{H}_{2} \mathrm{O}_{2}\right)$ e o radical hidroxil $\left(\mathrm{OH}^{\bullet}\right)(21,25,26)$.

\section{RADICAL SUPERÓXIDO $\left(\mathrm{O}_{2}^{-}\right)$}

$\mathrm{O}_{2}{ }^{-}$é um radical livre formado a partir da reação entre um elétron livre e uma molécula de $\mathrm{O}_{2}$, este é gerado principalmente durante a cadeia respiratória mitocondrial, e é o precursor da maioria das EROs $(21,23)$ (Figura 2).

$$
\mathbf{O}_{2}+\underset{\text { (eletron) }}{\bullet} \longrightarrow \mathbf{O}_{2}^{-}
$$

Figura 2. Reação entre uma molécula de oxigênio $\left(\mathrm{O}_{2}\right)$ e elétron livre promovendo a formação do radical superóxido $\left(\mathrm{O}_{2}^{-}\right)$.

A maior parte do $\mathrm{O}_{2}{ }^{-}$é produzido dentro da matriz mitocondrial, este radical livre é incapaz de atravessar as membranas lipídicas e tem vida curta sendo rapidamente convertido, por ação enzimática, à $\mathrm{H}_{2} \mathrm{O}_{2}(26-28)$.

\section{PERÓXIDO DE HIDROGÊNIO $\left(\mathrm{H}_{2} \mathrm{O}_{2}\right)$}

$\mathrm{O} \mathrm{H}_{2} \mathrm{O}_{2}$ é formado quando duas moléculas de $\mathrm{O}_{2}{ }^{-}$reagem com dois $\mathrm{H}^{+}$. Esta reação pode ocorrer espontaneamente ou catalisada por ação da enzima superóxido dismutase (SOD), comumente encontrada nas células dos mamíferos $(21,23,29)$ (Figura 3).

$$
2 \mathrm{O}_{2}^{-}+2 \mathrm{H}^{+} \longrightarrow \mathrm{H}_{2} \mathrm{O}_{2}+\mathrm{O}_{2}
$$

Figura 3. Formação do peróxido de hidrogênio $\left(\mathrm{H}_{2} \mathrm{O}_{2}\right)$ a partir da reação entre dois íons superóxido $\left(\mathrm{O}_{2}^{-}\right)$e dois prótons de hidrogênio $\left(\mathrm{H}^{+}\right)$.

$\mathrm{O} \mathrm{H}_{2} \mathrm{O}_{2}$ formado por dismutação do $\mathrm{O}_{2}^{-}$, por possuir estabilidade em sua estrutura, não é considerado propriamente um radical livre, entretanto é precursor na formação de uma potente ERO, $\mathrm{o}^{\bullet}$, além disto, $\mathrm{o}_{2} \mathrm{O}_{2}$ possui meia-vida longa e capacidade de se difundir pelas membranas biológicas $(7,23,30,31)$.

\section{RADICAL HIDROXIL (OH•)}

$\mathrm{O} \mathrm{OH}^{\bullet}$ é considerado um dos mais potentes agentes oxidantes, sendo capaz de atravessar membranas e reagir com moléculas tais como lipídeos insaturados das membranas e DNA. Este é formado a partir do $\mathrm{H}_{2} \mathrm{O}_{2}$ e sua estrutura é composta por um átomo de hidrogênio ligado a um átomo de oxigênio, onde um dos elétrons do $\mathrm{O}_{2}$ encontra-se desemparelhado (23).

Este radical é formado quando uma molécula de $\mathrm{H}_{2} \mathrm{O}_{2}$ recebe um elétron livre, decorrendo de reações envolvendo o íon férrico $\left(\mathrm{Fe}^{2+}\right)$ ou íon cobre $\left(\mathrm{Cu}^{+}\right)$, denominada reação de Fenton (7,21) (Figura 4), ou quando um $\mathrm{H}_{2} \mathrm{O}_{2}$ recepta um elétron proveniente do $\mathrm{O}_{2}^{-}$, denominada reação de Haber-Weiss $(7,30)$ (Figura 5). 


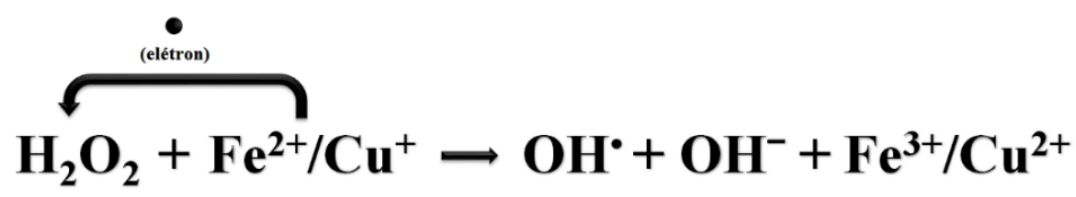

Figura 4. Reação de Fenton.

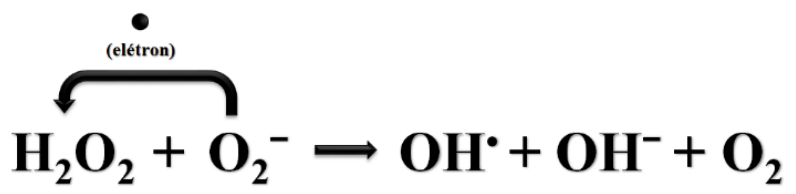

Figura 5. Reação de Haber-Weiss.

\section{PEROXIDAÇÃO LIPÍDICA}

As células espermáticas dos mamíferos contêm em sua membrana uma concentração elevada de ácidos graxos polinsaturados, embora a presença de elevadas concentrações destes ácidos graxos garanta maior fluidez e flexibilidade à membrana do espermatozoide, também a torna mais susceptível à ação deletéria das EROs, que são capazes de desencadear uma sequência de reações resultando, por vezes, em lesão irreversível à membrana $(6,30,32)$.

$\mathrm{O}$ processo de peroxidação lipídica decorre principalmente da ação do $\mathrm{OH}^{\bullet}$, que ao sequestrar um $\mathrm{H}^{+}$de um ácido graxo polinsaturado pertencente à bicamada fosfolipídica das membranas celulares, promove a desestabilização deste formando uma região de instabilidade, o radical alquil $\left(\mathrm{R}^{\bullet}\right)$, possuindo um elétron despareado. Este por sua vez, reage com uma molécula de $\mathrm{O}_{2}$ podendo formar outros dois radicais, alcoxil ( $\mathrm{RO}^{\bullet}$ ) ou peroxil $\left(\mathrm{ROO}^{\bullet}\right)$, que tendem a se estabilizar retirando uma molécula de $\mathrm{H}^{+}$e um elétron pertencentes aos fosfolipídeos adjacentes, formando hidroperóxido lipídico $(\mathrm{ROOH})$ e levando a formação de um novo $\mathrm{R}^{\bullet}$, promovendo uma reação em cadeia $(30,33)$ (Figura 6 ).

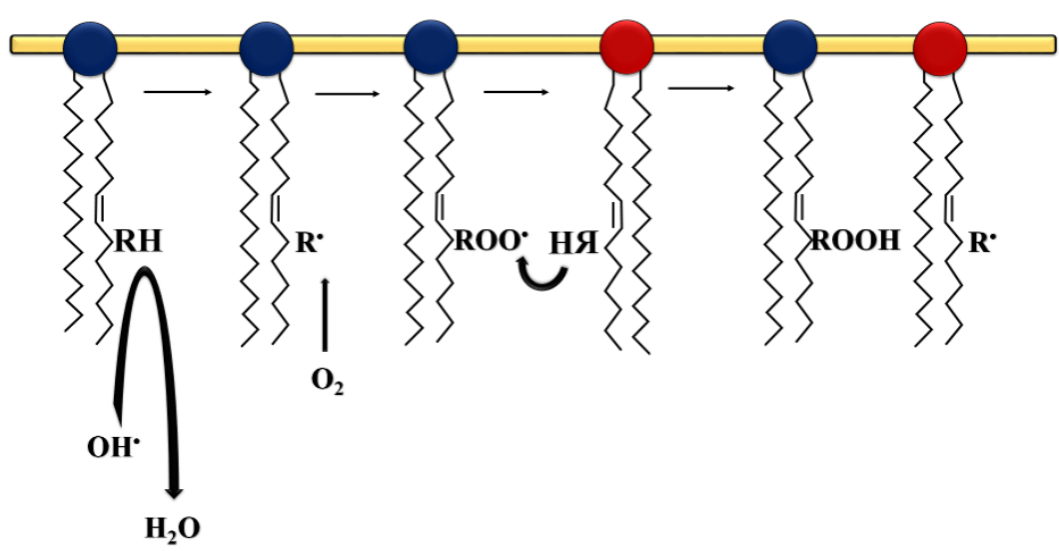

Figura 6. Esquematização da reação em cadeia ocorrida durante a peroxidação lipídica.

Caso esta sequência de reações não seja interrompida, pode resultar na comunicação entre duas regiões de instabilidade $\left(\mathrm{R}^{\bullet}-\mathrm{R}^{\bullet}, \mathrm{R}^{\bullet}-\mathrm{ROO}^{\bullet}\right.$ ou $\left.\mathrm{ROO} 0^{\bullet}-\mathrm{ROO} \mathrm{O}^{\bullet}\right)$ e esta reação é incompatível com a manutenção da estrutura da membrana afetando sua fluidez, elasticidade e permeabilidade, resultando em perda de viabilidade celular (34).

Os íons metálicos de ferro e cobre, também são capazes de desencadear a peroxidação lipídica, podendo doar ou sequestrar elétrons dos ROOH promovendo desestabilização do ácido graxo reiniciando a reação em cadeia (33) (Figura 7). 

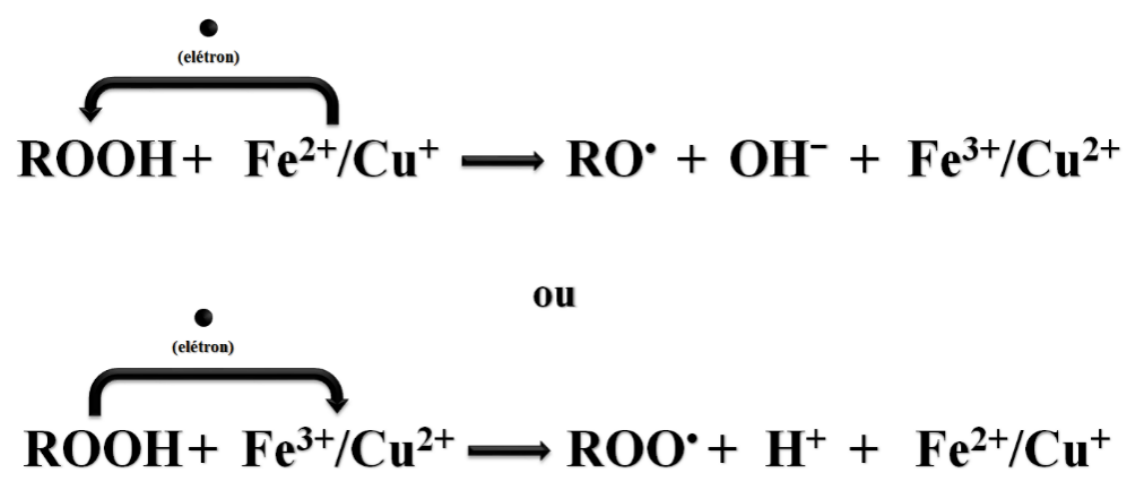

Figura 7. Participação dos íons metálicos $\left(\mathrm{Fe}^{2+} / \mathrm{Cu}^{+} \mathrm{ou} \mathrm{Fe}^{3+} / \mathrm{Cu}^{2+}\right)$ da desestabilização do hidroperóxido lipídico $(\mathrm{ROOH})$ e desencadeamento da peroxidação lipídica.

\section{CRIOINJÚRIAS}

Durante o processo de criopreservação os espermatozóides são submetidos a injúrias decorrente do estresse térmico, o que promove alterações funcionais e estruturais às células, podendo resultar em queda na motilidade e comprometimento da capacidade de fertilizar (26).

Sugere-se que durante a preservação pelo frio as mitocôndrias podem sofrer danos, levando ao comprometimento do metabolismo energético dos espermatozoides e aumento na produção de EROs, sendo relacionado com redução na cinética espermática e maior fragmentação de DNA (35).

Além disto, a maior ocorrência de peroxidação lipídica, resultante da elevação nos níveis de EROs, é considerada um dos principais danos causados pela criopreservação por promover desestruturação e aumento da permeabilidade da membrana, sendo indicado como um importante causador de danos subletais às células espermáticas nos equinos $(3,36)$. Observou-se que quando há o aumento da produção de peróxidos lipídicos ( $\mathrm{R}^{\bullet}$ ou $\left.\mathrm{ROO}^{\bullet}\right)$ ocorre uma queda na motilidade espermática, reduzindo a capacidade da fertilização (25).

Portanto a incorporação de agentes antioxidantes aos meios diluidores durante a refrigeração e congelação espermática é indicado como uma importante ferramenta para reduzir os danos decorrentes da elevação nos níveis de EROs resultantes do processo de criopreservação.

\section{PRINCIPAIS ANTIOXIDANTES E SUAS FUNÇÕES}

Durante o metabolismo oxidativo que ocorre nas mitocôndrias, cerca de 0,1 a $4 \%$ do $\mathrm{O}_{2}$ utilizado na respiração promovem a formação de $\mathrm{O}_{2}{ }^{-}$, quantidade mais que suficiente para induzir efeitos deletérios às células, necessitando que estes radicais sejam rapidamente neutralizados por agentes antioxidantes (20).

Para tal, as células espermáticas possuem compostos com função de combater o excesso de EROs e manter a homeostase celular, além disto são amplamente adicionadas aos meios diluidores de sêmen, substâncias naturais e sintéticas com funções antioxidantes (25).

Os antioxidantes nas células espermáticas agem basicamente de duas maneiras, eles podem impedir a produção das EROs ou interromper a reação em cadeia que ocorre durante peroxidação lipídica (37), podendo ser definidos como enzimáticos, quando sua função antioxidante é desempenhada por enzimas, e não enzimáticos (38). 


\section{ANTIOXIDANTES ENZIMÁTICOS}

Os antioxidantes enzimáticos compõem o primeiro sistema de defesa a agir nas células, neutralizando o excesso de EROs e prevenindo danos à estrutura celular. Fazem parte deste grupo, principalmente, a superóxido dismutase (SOD), catalase (CAT) e glutationa peroxidase (GPx) que em ação conjunta convertem $\mathrm{O}_{2}{ }^{-}$em $\mathrm{H}_{2} \mathrm{O}(27,39)$.

\section{SUPERÓXIDO DISMUTASE}

A SOD é um antioxidante enzimático encontrado na matriz mitocondrial, no citosol e no plasma seminal, tem a função de converter duas moléculas de $\mathrm{O}_{2}{ }^{-} \mathrm{em} \mathrm{H}_{2} \mathrm{O}_{2}$ e $\mathrm{O}_{2}$, por meio de reação de dismutação (29) (Figura 8).

$$
2 \mathrm{O}_{2}^{-}+2 \mathrm{H}^{+} \stackrel{\text { SOD }}{\longrightarrow} \mathrm{H}_{2} \mathrm{O}_{2}+\mathrm{O}_{2}
$$

Figura 8. Reação de formação do peróxido de hidrogênio $\left(\mathrm{H}_{2} \mathrm{O}_{2}\right)$ catalisada pela enzima superóxido dismutase (SOD).

Segundo Kankofer et al. (40), foi encontrada significativa atividade antioxidante no plasma seminal resultante da presença da enzima SOD. Foi observado também que a administração exógena de SOD ao ejaculado equino promoveu resultados significativo dos parâmetros de motilidade progressiva e integridade de membrana (9).

\section{GLUTATIONA PEROXIDASE}

Outra enzima amplamente encontrada nas células é a GPx, presente na matriz mitocondrial, espaço intermembranas e citosol. Sua função antioxidante é executada ao retirar $\mathrm{H}^{+}$e elétrons de duas moléculas de glutationa reduzida ( $\mathrm{GSH}$ ) doando-os ao $\mathrm{H}_{2} \mathrm{O}_{2}$, resultando em duas moléculas de $\mathrm{H}_{2} \mathrm{O}$ e uma de glutationa oxidada (GSSG) $(21,23)$ (Figura 9).

\section{$\mathrm{H}_{2} \mathrm{O}_{2}+2 \mathrm{GSH} \stackrel{\text { GPx }}{\longrightarrow} 2 \mathrm{H}_{2} \mathrm{O}+\mathrm{GSSG}$}

Figura 9. Ação da glutationa-peroxidase (GPx) na remoção do peróxido de hidrogênio $\left(\mathrm{H}_{2} \mathrm{O}_{2}\right)$, convertendo glutationa reduzida (GSH) em glutationa oxidada (GSSG).

A ação da GPx nos epermatozóides dos mamíferos é responsável por reduzir a ocorrência de peroxidação lipídica em virtude de sua capacidade em reduzir os níveis de $\mathrm{H}_{2} \mathrm{O}_{2}$ circulantes (41).

Oliveira et al. (10) em estudo com a espécie equina, observaram que a adição de 2,5 $\mathrm{mM}$ de glutationa peroxidase ao meio diluidor para congelação foi capaz de preservar motilidade total, motilidade progressiva, viabilidade e integridade da membrana plasmática, entretanto, concentrações superiores a $2,5 \mathrm{mM}$ deste antioxidante promoveram efeito deletério sobre os espermatozoides. 


\section{CATALASE}

A CAT também é responsável por decompor as moléculas de $\mathrm{H}_{2} \mathrm{O}_{2}$, convertendo-as a $\mathrm{H}_{2} \mathrm{O}$ e $\mathrm{O}_{2}$. Normalmente localiza-se nos peroxissomos, que são organelas presentes no citosol das células dos organismos eucariontes (38) (Figura 10).

\section{$2 \mathrm{H}_{2} \mathrm{O}_{2} \stackrel{\mathrm{CAT}}{\longrightarrow} 2 \mathrm{H}_{2} \mathrm{O}+\mathrm{O}_{2}$}

Figura 10. Conversão de peróxido de hidrogênio $\left(\mathrm{H}_{2} \mathrm{O}_{2}\right)$ à água $\left(\mathrm{H}_{2} \mathrm{O}\right)$ e oxigênio $\left(\mathrm{O}_{2}\right)$ por ação da catalase (CAT).

Apesar da comprovada função antioxidante representada pela CAT, Arich et al. (11), observaram que a inclusão de 1.8 unidades/L deste antioxidante ao meio diluidor para refrigeração de sêmen de equinos implicou em menores parâmetros de cinética espermática e integridade de membrana após 24 horas de refrigeração a $5^{\circ} \mathrm{C}$.

Já em estudo semelhante, Ball et al. (12) ao avaliarem a ação da CAT sobre o sêmen equino refrigerado a $5^{\circ} \mathrm{C}$ durante 72 horas, apesar de não terem observado melhores índices de motilidade espermática, evidenciaram maior manutenção da integridade acrossomal para as amostras acondicionadas na ausência do plasma seminal e tratadas com o antioxidante.

Em alguns casos, a linha primária de defesa contra as EROs, representada pelos antioxidantes enzimáticos, é insuficiente para promover redução satisfatória dos agentes oxidantes e homeostase celular aos espermatozoides, sendo necessário lançar mão da inclusão de antioxidantes não enzimáticos naturais ou sintéticos aos meios diluentes de sêmen (40).

\section{ANTIOXIDANTES NÃO ENZIMÁTICOS}

Os antioxidantes não enzimáticos são substâncias de baixo peso molecular que atuam como removedores dos agentes causadores dos danos oxidativos ou como reparadores da lesão já iniciada. Estes podem ser de origem natural, geralmente provenientes de vegetais, ou de origem sintética (42).

Dentre os antioxidantes não enzimáticos envolvidos na reprodução, destacam-se o ácido ascórbico (vitamina C), o $\alpha$-tocoferol (vitamina E), butil-hidroxitolueno (BHT) e a quercetina $(6,12,16,42)$.

\section{VITAMINA E ( $\alpha$-TOCOFEROL)}

A vitamina $\mathrm{E}$ ou $\alpha$-tocoferol $\left(\mathrm{C}_{29} \mathrm{H}_{50} \mathrm{O}_{2}\right)$ (Figura 11), é um poderoso antioxidante, lipossolúvel, presente na membrana interna das mitocôndrias, com a função de doar elétrons e $\mathrm{H}^{+}$ao $\mathrm{ROO}^{\bullet}$, interrompendo a reação em cadeia durante a peroxidação lipídica (31) (Figura 12).<smiles>Cc1c(C)c2c(c(C)c1O)CCC(C)(CCCC(C)CCCC(C)CCCC(C)C)O2</smiles>

Figura 11. Estrutura molecular do $\alpha$-tocoferol. 


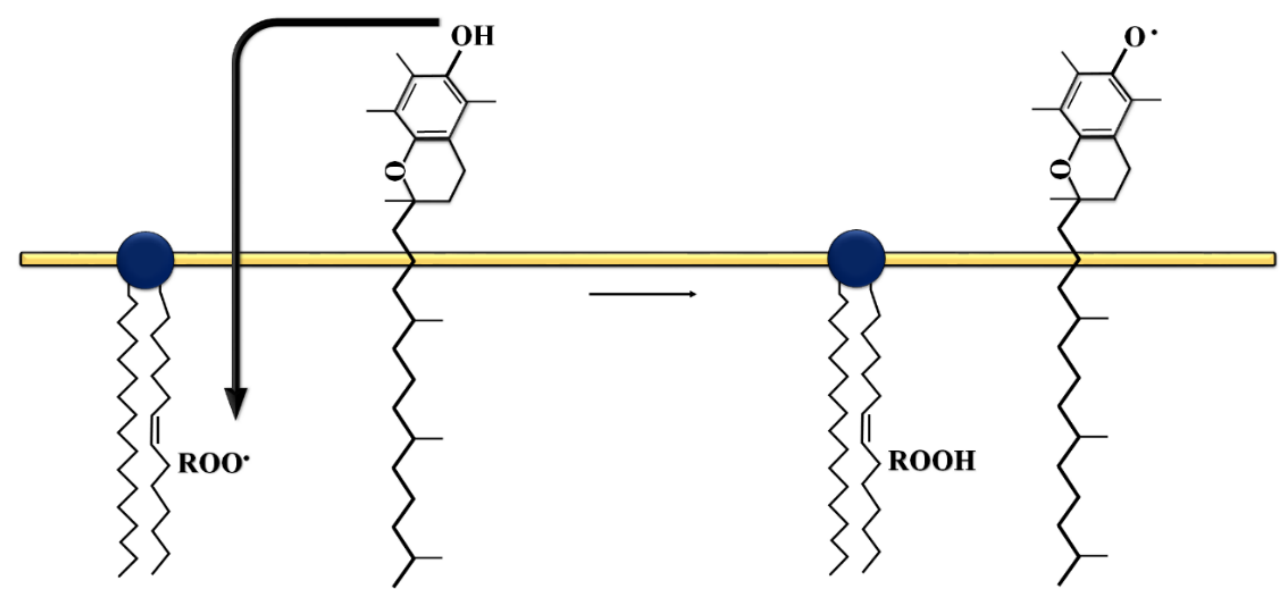

Figura 12. Mecanismo de ação do $\alpha$-tocoferol estabilizando o radical peroxil interrompendo a peroxidação lipídica.

Em estudo de Yousefian et al. (15), não foi notado efeito positivo da inclusão da vitamina $E$ sobre a cinética espermática, integridade de membranas ou lipoperoxidação durante a refrigeração do sêmen equino.

Já Almeida e Ball (13), observaram que a inclusão do $\alpha$-tocoferol no sêmen fresco de garanhões resultou em maior número de espermatozoides com motilidade progressiva sem que houvesse redução da peroxidação lipídica, entretanto quando as amostras foram submetidas à refrigeração a $5^{\circ} \mathrm{C}$ por 48 horas, a inclusão do antioxidante resultou em menores índices de peroxidação lipídica.

Resultados semelhantes foram encontrados por Franco et al. (14) em estudo com sêmen equino congelado, evidenciando que a incorporação de $\alpha$-tocoferol ao meio diluidor promoveu menores índices de peroxidação lipídica e maior viabilidade celular.

\section{VITAMINA C (ÁCIDO ASCÓRBICO)}

Outro importante antioxidante não enzimático é a vitamina $\mathrm{C}$ ou ácido ascórbico $\left(\mathrm{C}_{6} \mathrm{H}_{8} \mathrm{O}_{6}\right)$ (Figura 13). Esta funciona como agente redutor doando elétrons e $\mathrm{H}^{+}$às EROs, convertendo-as em espécies inofensivas e devido ao seu caráter hidrossolúvel, é incapaz de debelar diretamente a peroxidação lipídica, agindo em associação com a vitamina E para executar tal função (43) (Figura 14).

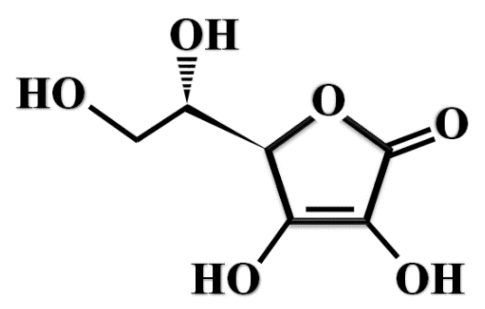

Figura 13. Estrutura molecular do ácido ascórbico. 


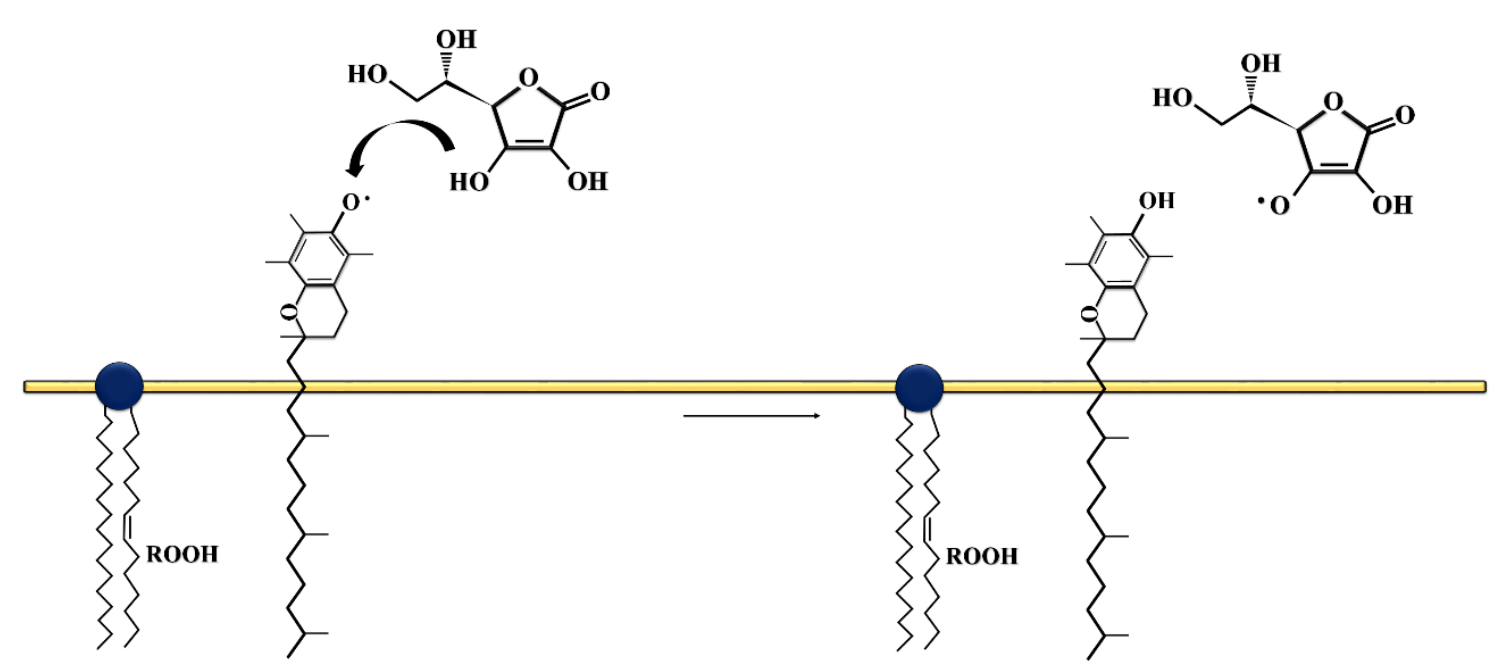

Figura 14. Ácido ascórbico promovendo a estabilização do $\alpha$-tocoferol durante a peroxidação lipídica.

A inclusão de ácido ascórbico no meio diluidor de refrigeração para sêmen equino, na concentração de $0,9 \mathrm{~g} / \mathrm{L}$, para as amostras mantidas à $5^{\circ} \mathrm{C}$ durante 24,48 e até 72 horas, resultou em melhores índices de integridade de membrana quando comparado aos grupos desprovidos deste antioxidante (11).

Em contrapartida, é sugerido que a vitamina $C$ também apresente uma função próoxidante, sendo capaz de doar elétrons aos íons metálicos $\mathrm{Fe}^{3+}$ e $\mathrm{Cu}^{2+}$, tornando-os compostos metálicos reduzidos, que vão participar da reação de Fenton, convertendo $\mathrm{H}_{2} \mathrm{O}_{2}$ à $\mathrm{OH}^{\bullet}$, imprimindo danos às biomoléculas (16,31) (Figura 15).
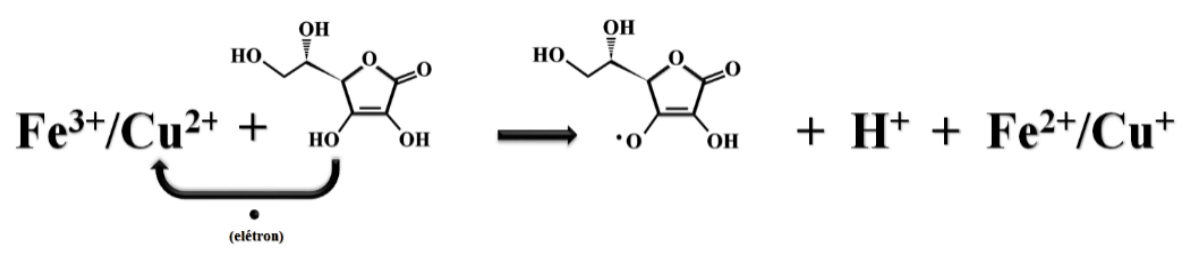

Figura 15. Esquematização da função pró-oxidante do ácido ascórbico, doando elétrons aos íons metálicos causadores da reação de Fenton.

Em estudos com sêmen congelado de garanhões, embora a inclusão de concentrações baixas deste antioxidante não tenha apresentado impacto sobre a viabilidade celular, elevadas concentrações resultaram em maior ocorrência de peroxidação lipídica e promoção de instabilidade à membrana plasmática (14).

\section{COMPOSTOS FENÓLICOS}

Entre os antioxidantes não enzimáticos, os compostos fenólicos têm significativa importância. Estes são caracterizados por possuir em sua estrutura um ou mais anéis aromáticos ligados a grupamentos hidroxila $(\mathrm{OH})$. Neste grupo de compostos destacam-se o butil-hidroxitolueno e a quercetina, sendo considerados antioxidantes mais eficientes do que as vitaminas $\mathrm{E}$ e $\mathrm{C}(44)$. 


\section{BUTIL-HIDROXITOLUENO (BHT)}

O BHT $\left(\mathrm{C}_{15} \mathrm{H}_{24} \mathrm{O}\right)$ (Figura 16) é um antioxidante sintético do grupo dos fenóis, análogo da vitamina $\mathrm{E}$, que está envolvido principalmente na reação de conversão dos radicais peroxil em hidroperóxidos lipídicos, doando $\mathrm{H}^{+}$e elétrons e interrompendo a reação em cadeia durante a peroxidação lipídica, sendo considerado desta maneira um eficiente protetor de membrana (45) (Figura 17).<smiles>Cc1cc(C(C)(C)C)c(O)c(C(C)(C)C)c1</smiles>

Figura 16. Estrutura molecular do butil-hidroxitolueno (BHT).

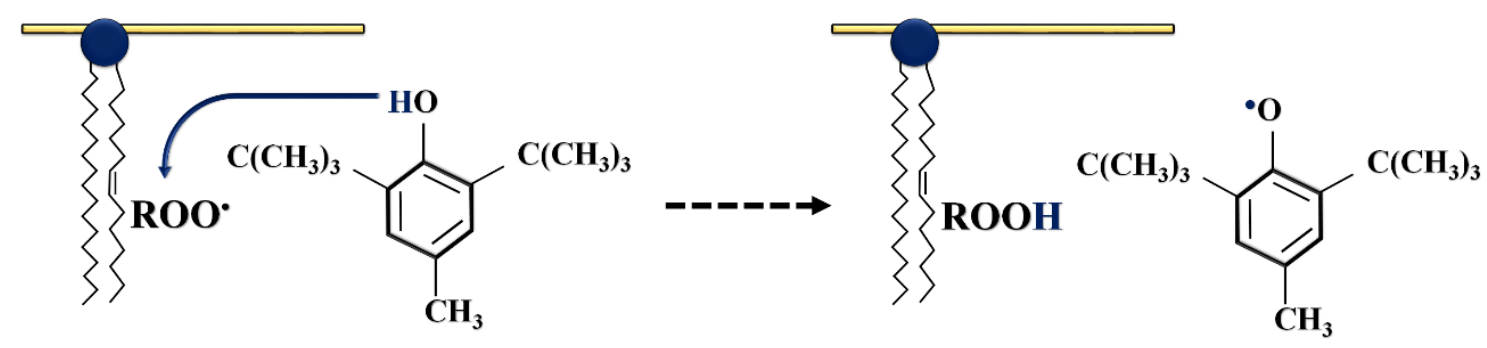

Figura 17. Mecanismo de ação do butil-hidroxitolueno (BHT) interrompendo a reação em cadeia ocorrida durante a peroxidação lipídica.

O BHT foi testado em sêmen de búfalos (46), touros (47), cães (48) e suínos (49) demonstrando aumento na motilidade, integridade de membrana plasmática e viabilidade espermática. Desta forma o BHT mostra-se uma opção promissora para reduzir os danos causados pelas EROs e promover maior longevidade aos espermatozoides, no entanto, há poucos estudos avaliando-se a influência do BHT no sêmen de equino. Ball et al. (12) não obtiveram resultados favoráveis com a inclusão de BHT em meio diluidor para refrigeração de sêmen equino. Morillo-Rodríguez et al. (2), em estudo com sêmen congelado de garanhões, também não encontraram efeito significativo da inclusão de $1 \mathrm{mM}$ de BHT no meio, não interferindo nos parâmetros de cinéticas espermática ou integridade das membranas.

\section{QUERCETINA}

A quercetina $\left(3,3^{\prime}, 4^{\prime}, 5,7\right.$-pentahidroxiflavona) é um antioxidante natural não enzimático, do grupo dos flavonoides, amplamente encontrado nas frutas, legumes, sementes, nozes e vinho tinto (28). Seu arranjo molecular é caracterizado como um polifenol flavonoide, possuindo cinco grupamentos $\mathrm{OH}$ em sua estrutura (50) (Figura 18). 
<smiles>O=c1c(O)c(-c2ccc(O)c(O)c2)oc2cc(O)cc(O)c12</smiles>

Figura 18. Estrutura molecular da quercetina.

O potencial antioxidante dos compostos flavonóides está diretamente ligado à quantidade de $\mathrm{OH}$ presentes em sua estrutura. Quanto mais grupamentos $\mathrm{OH}$ um flavonoide possui maior sua capacidade de doar elétrons e $\mathrm{H}^{+}(31,50)$. Os flavonoides monoidroxilados possuem baixa capacidade antioxidante, já os flavonoides com múltiplas $\mathrm{OH}$, como é o caso da quercetina, são considerados eficientes inativadores de EROs (31).

Em virtude da grande quantidade de $\mathrm{OH}$ encontradas em sua estrutura, a quercetina possui eficiente capacidade antioxidante, possibilitando a doação de até cinco elétrons e $\mathrm{H}^{+}$às EROs, estabilizando-as (37).

A quercetina pode promover sua ação antioxidante de três maneiras, reagindo com os radicais $\mathrm{O}_{2}^{-}$e $\mathrm{OH}^{\bullet}$, agindo como quelante de íons metálicos ou interrompendo a continuidade da peroxidação lipídica (51).

De acordo com Chen et al. (52), este flavonoide é capaz de reduzir consideravelmente as concentrações de $\mathrm{O}_{2}^{-}$, além de possuir a capacidade de reagir diretamente com o $\mathrm{OH}^{\bullet}$, doando elétrons e $\mathrm{H}^{+}$para este radical resultando na formação de $\mathrm{H}_{2} \mathrm{O}(43,52)$ (Figura 19).

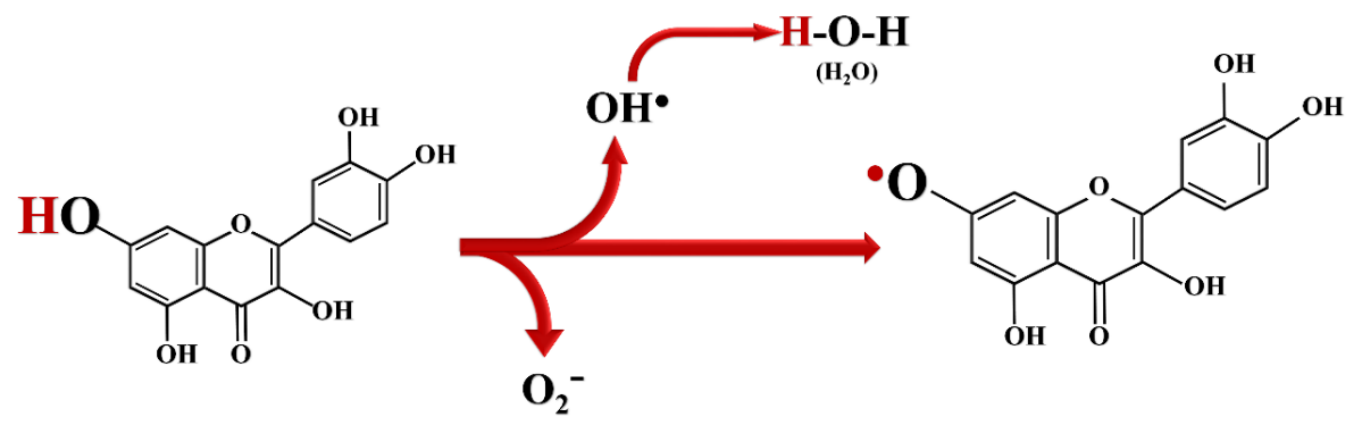

Figura 19. Ação da quercetina estabilizando os radicais superóxido $\left(\mathrm{O}_{2}^{-}\right)$ou radical hidroxil $\left(\mathrm{OH}^{\bullet}\right)$.

Este antioxidante também tem a capacidade de atuar como quelante de íons metálicos, reagindo e impedindo que o íon $\mathrm{Fe}^{2+}$ doe elétrons para $\mathrm{o}_{2} \mathrm{O}_{2}$ interrompendo a reação de Fenton e formação do $\mathrm{OH}^{\bullet}$ (53). Além de minimizar também a participação deste íon metálico no desencadeamento direto da peroxidação lipídica (33) (Figura 20). 


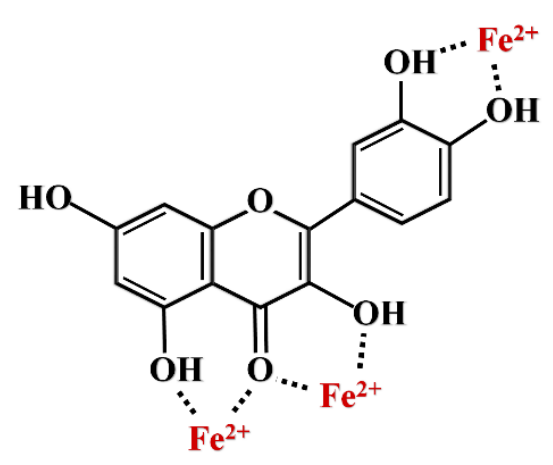

Figura 20. Esquematização da função da quercetina como quelante do íon férrico $\left(\mathrm{Fe}^{2+}\right)$.

Outra possibilidade de ação antioxidante realizada pela quercetina durante a peroxidação lipídica ocorre em decorrência deste flavonoide possuir caráter lipofílico, sendo capaz de interagir com a bicamada lipídica e permitindo a doação direta de $\mathrm{H}^{+}$e elétrons a radicais como o $\mathrm{ROO}^{\bullet}$, estabilizando e convertendo-os a $\mathrm{ROOH}$, impedindo assim a continuidade da reação em cadeia $(31,51)$ (Figura 21).
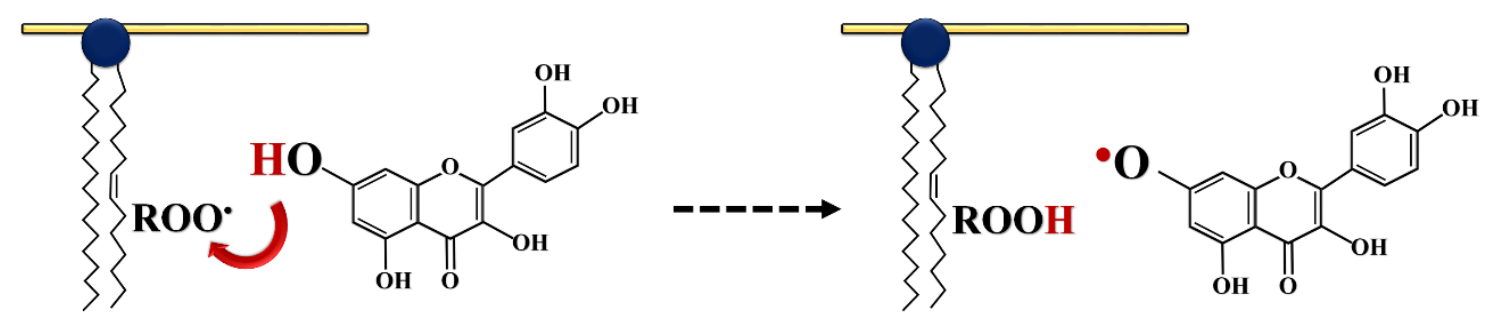

Figura 21. Bloqueio à reação em cadeia durante a peroxidação lipídica por ação da quercetina, ao doar um elétron e um próton $\left(\mathrm{H}^{+}\right)$ao radical peroxil $\left(\mathrm{ROO}^{\bullet}\right)$ formando hidroperóxido lipídico $(\mathrm{ROOH})$.

A adição de $0,1 \mathrm{mM}$ de quercetina ao meio de refrigeração para sêmen de equinos mantido à $5^{\circ} \mathrm{C}$ por até 144 horas resultou em maior preservação da motilidade espermática total, maior integridade de membrana, maiores níveis intracelulares de ATP e menor peroxidação lipídica (16).

Gibb et al. (17), em estudo com utilização da quercetina no sêmen congelado de equinos observaram que a inclusão do antioxidante promoveu redução nos níveis de fragmentação de DNA e aumento da motilidade total dos espermatozoides.

\section{CONSIDERAÇÕES FINAIS}

Embora o avanço na utilização do sêmen criopreservado de garanhões promova cada vez mais a necessidade de inclusão de antioxidantes nos meios diluidores, ainda há resultados controversos com relação à eficácia de determinados agentes antioxidantes dentro da espécie, necessitando-se de aprofundamento sobre as interações das substancias com as células espermáticas e suas possíveis consequências.

\section{AGRADECIMENTOS}

À Fundação de Amparo à Pesquisa do Estado de São Paulo (FAPESP), pela concessão do auxílio à pesquisa (Processo no 2014/01681-4). 


\section{REFERÊNCIAS}

1. Nouri H, Towhidi A, Zhandi M, Sadeghi R. The effects of centrifuged egg yolk used with INRA plus soybean lecithin extender on semen quality to freeze miniature caspian horse semen. J Equine Vet Sci. 2013;33:1050-3.

2. Morillo-Rodríguez A, Macías-García B, Tapia JA, Ortega-Ferrusola C, Peña FJ. Consequences of butylated hydroxytoluene in the freezing extender on post-thaw characteristics of stallion spermatozoa in vitro. Andrologia. 2012;44:688-95.

3. Ortega-Ferrusola C, Gonzalez FL, Morrell JM, Salazar SC, Macías-García B, RodriguezMartinez H, et al. Lipid peroxidation, assessed with BODIPY-C11, increases after cryopreservation of stallion spermatozoa, is stallion-dependent and is related to apoptoticlike changes. Reproduction. 2009;138:55-63.

4. García BM, Fernández LG, Ferrusola CO, Rodríguez AM, Bolaños JMG, Martinez HR, et al. Fatty acids and plasmalogens of the phospholipids of the sperm membranes and their relation with the post-thaw quality of stallion spermatozoa. Theriogenology. 2011;75:81118.

5. Aitken RJ, Harkiss D, Knox W, Paterson M, Irvine DS. A novel signal transduction cascade in capacitating human spermatozoa characterised by a redox-regulated, cAMPmediated induction of tyrosine phosphorylation. J Cell Sci. 1998;111:645-56.

6. Lenzi A, Gandini L, Lombardo F, Picardo M, Maresca V, Panfili E, et al. Polyunsaturated fatty acids of germ cell membranes, glutathione and blutathione-dependent enzymePHGPx: from basic to clinic. Contraception. 2002;65:301-4.

7. Ford WCL. Regulation of sperm function by reactive oxygen species. Hum Reprod Update. 2004;10:387-99.

8. Kothari S, Thompson A, Agarwal A, Du Plessis SS. Free radicals: their beneficial and detrimental effects on sperm function. Indian J Exp Biol. 2010;48:425-35.

9. Cocchia N, Pasolini MP, Mancini R, Petrazzuolo O, Cristofaro I, Rosapane I, et al. Effect of sod (superoxide dismutase) protein supplementation in semen extenders on motility, viability, acrosome status and ERK (extracellular signal-regulated kinase) protein phosphorylation of chilled stallion spermatozoa. Theriogenology. 2011;75:1201-10.

10. Oliveira RA, Wolf CA, Oliveira MA, Gambarini ML. Addition of glutathione to na extender for frozen equine sêmen. J Equine Vet Sci. 2013;33:1148-52.

11. Aurich $\mathrm{JE}_{2}$ Schönherr $\mathrm{U}_{2}$ Hoppe $\mathrm{H}_{2}$ Aurich C. Effects of antioxidants on motility and membrane integrity of chilled-stored stallion semen. Theriogenology. 1997;48:185-92.

12. Ball BA, Medina V, Gravance CG, Baumber J. Effect of antioxidants on preservation of motility,viability and acrosomal integrity of equine spermatozoa during storage at $5^{\circ} \mathrm{C}$. Theriogenology. 2001;56:577-89. 
13. Almeida J, Ball BA. Effect of $\alpha$-tocopherol and tocopherol succinate on lipid peroxidation in equine spermatozoa. Anim Reprod Sci. 2005;87:321-37.

14. Franco JSV, Chaveiro A, Góis A, Silva FMS. Effects of $\alpha$-tocopherol and ascorbic acid on equine semen quality after cryopreservation. J Equine Vet Sci. 2013;33:787-93.

15. Yousefian I, Zare-Shahneh A, Zhandi M. The effect of coenzyme q10 and $\alpha$-tocopherol in skim milk-based extender for preservation of caspian stallion semen in cool condition. J Equine Vet Sci. 2014;34:949-54.

16. McNiven MA, Richardson GF. Chilled storage of stallion semen using perfluorochemicals and antioxidants. Cell Preserv Technol. 2003;1:165-74.

17. Gibb Z, Butler TJ, Morris LH, Maxwell WM, Grupen CG. Quercetin improves the postthaw characteristics of cryopreserved sex-sorted and nonsorted stallion sperm. Theriogenology. 2013;79:1001-9.

18. Varner DDD, Gibb Z, Aitken RJ. Stallion fertility: a focus on the spermatozoon. Equine Vet J. 2015;47:16-24.

19. Varner D, Johnson L. From a sperm's view - revisiting our perception of this intriguing cell. AEEP Proceedings. 2007;53:104-77.

20. Nelson DL, Cox MM. Lehninger princípios da bioquímica. 6a ed. Porto Alegre: Artmed; 2014.

21. Venditti P, Di Stefano L, Di Meo S. Mitochondrial metabolism of reactive oxygen species. Mitochondrion. 2013;13:71-82.

22. Lenaz G, Fato R, Formiggini G, Genova ML. The role of coenzyme Q in mitochondrial electron transport. Mitochondrion. 2007;7:S8-33.

23. Turrens JF. Mitochondrial formation of reactive oxygen species. J Physiol. 2003;552:335-44.

24. Kussmaul L, Hirst J. The mechanism of superoxide production by NADH: ubiquinone oxidoreductase (complex I) from bovine heart mitochondria. Proc Natl Acad Sci U S A. 2006;103:7607-12.

25. Lenzi A, Gandini L, Picardo M, Tramer F, Sandri G, Panfili E. Lipoperoxidation damage of spermatozoa polyunsaturated fatty acids (PUFA): scavenger mechanisms and possible scavenger therapies. Front Biosci. 2000;5:1-15.

26. Maia MS, Bicudo SD. Radicais livres, antioxidantes e função espermática em mamíferos: uma revisão. Rev Bras Reprod Anim. 2009;33:183-93.

27. Lenaz G. The mitochondrial production of reactive oxygen species: mechanisms and implications in human pathology. IUBMB Life. 2001;52:159-64. 
28. Nogueira BG, Bitencourt JL, Sampaio BFB, Bender ESC, Costa e Silva EV, Zúccari CESN. Peroxidação lipídica e agentes antioxidantes no sêmen de mamíferos. Rev Eletronica Vet. 2013;15:1-15.

29. Lambert AJ, Brand MD. Superoxide production by NADH: ubiquinone oxidoreductase (complex I) depends on the $\mathrm{pH}$ gradient across the mitochondrial inner membrane. Biochem J. 2004;382:511-7.

30. Griveau JF, Le Lannou D. Reactive oxygen species and human spermatozoa: physiology and pathology. Int J Androl. 1997;20:61-9.

31. Barreiros ALBS, David JM, David JP. Estresse oxidativo: relação entre geração de espécies reativas e defesa do organismo. Quim Nova. 2006;29:113-23.

32. Salmani H, Nabi MM, Vaseghi-Dodaran H, Rahman MB, Mohammadi-Sangcheshmeh A, Shakeri M, et al. Effect of glutathione in soybean lecithin-based semen extender on goat semen quality after freeze-thawing. Small Rumin Res. 2013;112:123-7.

33. Halliwell B. Oxidative stress and neurodegeneration. Where are we now? J Neurochem. 2006;97:1634-58.

34. Catala A. An overview of lipid peroxidation with emphasis in outer segments of photoreceptors and the chemiluminescence assay. Int $\mathrm{J}$ Biochem Cell Biol. 2006;38:1482-95.

35. Schober D, Aurich C, Nohl H, Gille L. Influence of cryopreservation on mitochondrial functions in equine spermatozoa. Theriogenology. 2007;68:745-54.

36. Alvarez JG, Storey BT. Evidence for increased lipid peroxidative damage and loss of superoxide dismutase activity as a mode of sublethal cryodamage to human sperm during cryopreservation. J Androl. 1992;13:232-41.

37. Silva ECB, Guerra MMP. Terapias antioxidantes na criopreservação espermática. Rev Port Cienc Vet. 2012;107:143-9.

38. Andreyev AY, Kushnareva YE, Starkov AA. Mitochondrial metabolism of reactive oxygen species. Biochemistry. 2005;70:200-14.

39. Nogueira BG, Bitencourt JL, Sampaio BFB, Bender ESC, Costa e Silva EV, Zúccari CESN. Peroxidação lipídica e agentes antioxidantes no sêmen de mamíferos. Rev Eletronica Vet. 2013;15:1-15.

40. Kankofer M, Kolm G, Aurich J, Aurich C. Activity of glutathione peroxidase, superoxide dismutase and catalase and lipid peroxidation intensity in stallion semen during storage at $5^{\circ} \mathrm{C}$. Theriogenology. 2005;63:1354-65.

41. Alvarez G, Storey BT. Role of glutathione peroxidase in protecting mammalian spermatozoa from loss of motility caused by spontaneous lipid peroxidation. Gamete Res. 1989;23:77-90. 
42. Andrade ER, Melo-Sterza FA, Seneda MM, Alfieri AA. Consequências da produção das espécies reativas de oxigênio na reprodução e principais mecanismos antioxidantes. Rev Bras Reprod Anim. 2010;34:79-85.

43. Wright JS, Johnson ER, Dilabio GA. Predicting the activity of phenolic antioxidants: theoretical method, analysis of substituent effects, and application to major families of antioxidants. J Am Chem Soc. 2001;123:1173-83.

44. Stojanovic S, Sprinz H, Brede O. Efficiency and mechanism of the antioxidant action of trans-resveratrol and its analogues in the radical liposome oxidation. Arch Biochem Biophys. 2001;391:79-89.

45. Aitken RJ, Clarkson JS. Cellular basis of defective sperm function and its association with the genesis of reactive oxygen species by human spermatozoa. J Reprod Fertil. 1988;81:459-69.

46. Ijaz A, Hussain A, Aleem M, Yousaf MS, Rehman H. Butylated hydroxytoluene inclusion in semen extender improves the post-thawed semen quality of Nili-Ravi buffalo (Bubalus bubalis). Theriogenology. 2009;71:1326-9.

47. Ansari MS, Rakha BR, Akhter S. Effect of butylated hydroxytoluene supplementation in extender on motility, plasmalemma and viability of sahiwal bull spermatozoa. Pak J Zool. 2011;43:311-4.

48. Neagu VR, García BM, Sandoval CS, Rodríguez AM, Ferrusola CO, Fernández LG, et al. Freezing dog semen in presence of the antioxidant butylated hydroxytoluene improves postthaw sperm membrane integrity. Theriogenology. 2010;73:645-50.

49. Trzcińska M, Bryła M, Gajda B, Gogol P. Fertility of boar semen cryopreserved in extender supplemented with butylated hydroxytoluene. Theriogenology. 2015;83:307-13.

50. Alrawaiq NS, Abdullah A. A review of flavonoid quercetin: metabolism, bioactivity and antioxidant properties. Int J PharmTech Res. 2014;6:933-41.

51. Afanas'ev IB, Dorozhko AI, Brodskii AV, Kostyuk VA, Potapovitch AI. Chelating and free radical scavenging mechanisms of inhibitory action of rutin and quercetin in lipid peroxidation. Biochem Pharmacol. 1989;38:1763-9.

52. Chen Y, Zheng R, Jia Z, Ju Y. Flavonoids as superoxide scavengers and antioxidants. Free Radic Biol Med. 1990;9:19-21.

53. Gülçin I. Antioxidant activity of food constituents: an overview. Arch Toxicol. 2012;86:345-91.

Recebido em: 30/08/2016

Aceito em: 31/05/2017 